\title{
LA NOUVELLE CARTE SCOLAIRE \\ DU CANTON DE VAUD \\ René Meylan
}

Avec une carte en couleurs

La collection des cartes scolaires cantonales de la Suisse vient de s'enrichir d'une nouvelle unité, la carte du Canton de Vaud, sortie des ateliers Kümmerly \& Frey, de Berne, éditée par la maison Payot \& Cie, de Lausanne, pour le département de l'Instruction publique du Canton de Vaud. Les $3209 \mathrm{~km}^{2}$ du Canton de Vaud sont inscrits, à l'échelle de 1:150000, dans un quadrilatère de $60 \mathrm{~cm}$. de largeur et $62 \mathrm{~cm}$. de hauteur. La caprice des frontières et l'éloignement des points extrêmes - $100 \mathrm{~km}$. de la frontière genevoise à l'embouchure de la Broye; $90 \mathrm{~km}$. de ce point au pont de St-Maurice - obligent à englober sur la carte de vastes étendues de territoires étrangers au Canton de Vaud. La carte, coupée en sa partie septentrionale à la latitude de St-Blaise, comprend ainsi la moitié du Canton de Neuchâtel. Au Sud est visible tout le pays qui s'étend dans liarc du Léman, de Genève à St-Maurice. A l'Est, du confluent de la Sarine et de l'Aar jusqu'à la Dent de Ruth, la carte englobe la majeure partie du canton de Fribourg et, à l'Ouest, un fragment important des plateaux comtois.

La nouvelle édition se distingue des précédentes par un remarquable effort de représentation du relief et l'on peut dire que la réussite est complète. La gamme des couleurs produit une impression très harmonieuse et les courbes de niveau, discrètement dessinées, modèlent cependant les formes avec toute la netteté désirable.

Pourquoi faut-il qu'en reproduisant la légende de l'édition précédente on ait maintenu «équidistance des courbes de niveau $100 \mathrm{~m} . »$, alors que cette équidistance est de $50 \mathrm{~m}$. pour l'édition de 1950 ? Et, puisque nous en arrivons à formuler/ une critique, nous nous bornerons, étant donné la place dont nous disposons, à quelques brèves observations. N'aurait-il pas été indiqué de profiter de cette occasion pour abandonner la cote de la Pierre du Niton? Il faudra s'y résoudre un jour ou l'autre; il est inutile d'attendre que la nouvelle carte nationale nous y habitue. Pourquoi a-t-on chargé de l'article certains noms de rivières, alors que d'autres ne l'ont pas? (Rhône, Orbe, Aubonne, Veveyse, etc., d'une part et la Sarine, la Thièle, la Broye, etc., d'autre part?) La nomenclature n'est pas toujours très heureuse pour une carte «scolaire». Un seul exemple: qu'apporte un nom absolument inusité, comme «Marcause », là où l'on voudrait lire Promenthoux ou Pointe de Promenthoux qui sert à délimiter le Petit-Lac? On peut déplorer aussi quelques absences: «La Givrine 》, point culminant des voies ferrées jurassiennes, «Le Day», bifurcation ferroviaire, "Tour de la Molière », lieu historique, il est vrai en terre fribourgeoise d'Estavayer, l'œil de l'Helvétie, d'où l'on voit loin à la ronde. La mise en évidence des routes à priorité, dont le réseau se dessine d'une manière très apparente, nous semble, par contre, une innovation intéressante.

Une carte nouvelle est toujours une promesse d'enrichissement. S'il s'agit d'une carte scolaire cantonale, au plaisir que l'on prend à la déployer s'ajoute la certitude que des milliers d'enfants y auront l'heureuse révélation de leur patrie. Cette patrie, ils la tiennent dans leurs mains, comme ils l'embrassent d'un coup d'œil, tout entière, du haut d'un des belvédères jurassiens. De la carte au paysage, ou du paysage à la carte, le passage se fait sans la moindre difficulté, à cette échelle où la carte parle encore par tous ses détails. Le Canton de Vaud a le privilège de s'étendre aux Alpes, au Plateau et au Jura, de sorte que sa carte scolaire présente les traits caractéristiques des trois grandes régions naturelles de la Suisse et cela lui confère un intérêt particulier. 
Der Aufsatz gibt eine Einführung in die vom Verlag Payot, Lausanne, verlegte und von der Firma Kümmerly \& Frey, Bern, gedruckte neue Waadtländer Schulkarte.

\title{
LA NUOVA CARTA SCOLASTICA DEL CANTON VAUD
}

L'autore fa una introduzione esplicativa alla nuova carta scolastica del Canton Vaud edita dalla Casa editrice Payot di Losanna e stampata dalla Firma Kümmerly \& Frey di Berna.

\section{L'ACTIVITÉ DE LA RECHERCHE SCIENTIFIQUE DANS LES PYRÉNÉES}

\author{
LE PREMIER CONGRÈS INTERNATIONAL \\ D'ÉTUDES PYRÉNÉENNES DE SAINT-SÉBASTIEN \\ $22-26$ SEPTEMBRE 1950
}

Henri Onde

\begin{abstract}
L'Instituto de Estudios Pirenaicos de Saragosse a attiré l'attention du monde savant par la publication de son remarquable périodique Pirineos (1). Cette revue, soutenue par le Consejo Superior de Investigaciones Científicas, s'est préoccupée d'emblée d'envisager le problème pyrénéen sous tous ses aspects, géologique, géographique, linguistique, historique etc., prouvant ainsi l'intérêt que prend l'Espagne aux études régionales (2). Or, 5 ans après la parution du premier numéro de Pirineos, nos amis espagnols ont été en mesure d'organiser à Saint-Sébastien, du 22 au 26 septembre 1950, le premier Congrès international d'Etudes pyrénéennes. L'Institut des Etudes pyrénéennes a été aidé dans son énorme tâche par la Junta de Relaciones Culturales du Ministère des Affaires Etrangères, par S. E. le Gouverneur Civil dE Gulpuzcon et par la Real Societad Vascongada de Amigos del Pais, mais s'il a remporté un si éclatant sucçès, il le doit pour une très large part à son Directeur, M. Luis Solf́: Sabaris, de l'Université de Barcelone, ainsi qu'à son Secrétaire, M. JosÉ MANuel CAsas Torres, de l'Université de Saragosse qui, par son activité, son rayonnement personnel, sert admirablement la cause de la géographie outre-Pyrénées.

Plusieurs semaines déjà avant le Congrès un questionnaire avait été distribué aux futurs participants, leur demandant notamment dans quel ordre d'urgence et avec quels moyens les études sur les Pyrénées doivent être entreprises, les priant de se prononcer sur le type de carte de base à utiliser pour représenter les résultats acquis et, enfin, de donner leur avis sur l'opportunité d'un Atlas des Pyrénées. Un dépouillement de toutes les réponses a pu figurer dans le fascicule imprimé contenant la liste des membres, des sections et des communications. Par un autre tour de force, les organisateurs du Congrès ont réussi à faire imprimer toutes les communications parvenues au Secrétaire dans les délais prescrits. Par ailleurs, le choix de Saint-Sébastien comme siège du Congrès s'est révélé particulièrement heureux. Cette capitale régionale, dont la population en 1948 était estimée à 120000 âmes, est par elle-même fort attachante. Le site de tombolo, les problèmes que posent la topographie littorale (3), la juxtaposition à la vieille cité maritime d'une ville balnéaire conçue suivant un plan harmonieux, tout ici constitue une vivante leçon de géographie urbaine qu'il est encore possible d'illustrer à l'aide de la belle collection de plans anciens, de cartes topographiques et marines exposées au Musée de San Telmo et au Musée Naval. Centre intellectuel, Saint-Sébastien a logé à l'aise, à l'Instituto Peñaflorida les nombreuses sections du Congrès et s'est montré assez riche en talents pour offrir à ses hôtes des concerts vocaux exécutés par trois chœurs différents et un festival de danses basques. Les organisateurs du premier Congrès international d'Etudes pyrénéennes se sont ingéniés par des réceptions, des excursions et des spectacles à distraire et à instruire leurs invités, et ceux-ci conserveront le durable souvenir de l'hospitalité délicate et généreuse qu'ils ont reçue partout où ils ont passé.

Le Congrès a réuni une centaine de participants, Espagnols et Français pour la plupart, mais qui comptaient entre autre des représentants du Brésil, du Portugal, de la Grande-Bretagne, de l'Allemagne, de l'Italie, de la Belgique et de la Suisse. Le travail s'y est réparti entre 6 sections: I. Géologie, Morphologie et Géophysique; II. Météorologie, Pédologie, Botanique et Zoologie; III. Préhistoire, Anthropologie et Ethnologie; IV. Géographie et Economie; V. Histoire, Art et Droit; VI. Philologie. En dehors de leurs sections respectives les congressistes ont assisté à la projection d'un film sur les grands travaux hydroélectriques en cours dans les Pyrénées espagnoles, ils ont entendu M. Henri Gaussen, de l'Université de Toulouse, commenter une magnifique série de photographies en couleurs sur le Roussillon et les Pyrénées françaises et M. Сн. Higoune't, de l'Université
\end{abstract}

